\title{
How Small-Sided and Conditioned Games Enhance Acquisition of Movement and Decision-Making Skills
}

\author{
Keith Davids ${ }^{1,2}$, Duarte Araújo ${ }^{3}$, Vanda Correia ${ }^{3,4}$, and Luís Vilar ${ }^{5,6}$ \\ ${ }^{1}$ School of Exercise and Nutrition Science, Queensland University of Technology, Queensland, Australia; \\ ${ }^{2}$ Faculty of Sport and Health Sciences, University of Jyväskylä, Jyväskylä, Finland; ${ }^{3}$ SpertLab, CIPER, Faculdade de \\ Motricidade Humana, Universidade Técnica de Lisboa, Lisbon; ${ }^{4}$ School of Education and Communication, \\ University of Algarve, Faro; ${ }^{5}$ Faculty of Physical Education and Sports, Lusófona University of Humanities \\ and Technologies, Lisbon, Portugal; and ${ }^{6}$ Faculdade de Motricidade Humana, Universidade Técnica de Lisboa, \\ Lisbon, Portugal
}

DAVIDS, K., D. ARAÚJO, V. CORREIA, and L. VILAR. How small-sided and conditioned games enhance acquisition of movement and decision-making skills. Exerc. Sport Sci. Rev., Vol. 41, No. 3, pp. 154-161, 2013. This article summarizes research from an ecological dynamics program of work on team sports exemplifying how small-sided and conditioned games (SSCG) can enhance skill acquisition and decision-making processes during training. The data highlighted show how constraints of different SSCG can facilitate emergence of continuous interpersonal coordination tendencies during practice to benefit team game players.

Key Words: ecological dynamics, interpersonal coordination, information-action coupling, skill acquisition, representative design

\section{INTRODUCTION}

A major interest in sports sciences has been the development of training programs that provide team sports coaches with reliable methods for improving training while enhancing player performance. Traditional application of science to team games has emphasized training of specific physiological fitness indices, perceptual skills, and technical or tactical actions through use of repetitive practice drills often performed in isolation from the competitive performance context (33). A large body of work, from an organism-centered perspective, has focused on benefits of increasing difficulty and adaptive uncertainty for individuals in learning environments through contextual interference effects and providing summary or bandwidth feedback. Organism-centered theories portray learning with these traditional methods as leading to an increasing enrichment of internalized movement representations (10).

\footnotetext{
Address for correspondence: Keith Davids, Ph.D., School of Exercise and Nutrition Science, Queensland University of Technology, Victoria Park Rd, Kelvin Grove, Queensland 4059, Australia (E-mail: k.davids@qut.edu.au).

Accepted for publication: February 18, 2013.

Associate Editor: Ryan E. Rhodes, Ph.D.
}

Traditional methods, such as part-task training and adaptive instructions $(41,42)$, are used extensively as coaching strategies in sports. Part-task training involves practicing subsets of task components as a precursor to performance of the whole task. Adaptive training occurs when difficulty is increased gradually as a task is mastered. Both instructional methods involve little sustained manipulation of practice task constraints, seeking to facilitate learners searching for unique performance solutions (25). Such traditional methods provide a limited scope for action variability in learners because a key aim of practitioners is to decrease uncertainty of actions and rationalize decisionmaking processes in training drills. Despite these reported benefits, traditional utilization of practice drills has been criticized because they neglect the active role of the performance environment in shaping movement behavior and decision making (10), sustaining a dysfunctional rupture in the performerenvironment relationship (cf. the concept of organismic asymmetry in sports science (9)).

In this article, we overview findings from our research on team games performance from an ecological dynamics perspective, which have implications for designing training environments. Based on these insights, we provide a rationale for considering the performer-environment scale of analysis in designing training tasks. A significant proposition from our program of work is that a training task design in team sports needs to represent the specifying variables (i.e., key informational 
sources) from a competitive performance environment that individuals use to regulate actions and make decisions. Our findings reveal how players and teams use specifying information available in the competitive environment to regulate coordinated activities and functional behaviors that support successful performance. These findings imply that practitioners need to design dynamic training simulations that capture the inherent variability of the competitive performance environment, leading performers to use information that is specifying (i.e., functionally relevant to support action) $(30,31)$.

\section{TRADITIONAL TRAINING METHODS IN TEAM SPORTS}

Traditionally, training in team sports has had a biased focus on the specific constraints of the individual, such as improving physiological indices, and mental representations of intrapersonal or interpersonal patterns of coordination (technical and tactical aspects, respectively) (9). Instead, our work suggests that training needs to be refocused on the performer-environment relationship (10). To exemplify, tasks often are designed for performance without opponents or with passive opposition to simplify decision making during repetitive drill practice under conditions of reduced uncertainty (25). This is because active opposition creates variability and uncertainty for the interpersonal interactions that emerge between players during training. Use of static markers may benefit repetition of discrete performance outcomes during practice, especially early in learning (3). These drills can enhance the development of speed, endurance, flexibility, power, and performance of specific technical actions by increasing the number of shots at goal, passes to teammates, dribbling sequences, and number of interceptions/ tackles made. For example, when coaching dribbling in soccer, learners are first taught to control the ball in isolation so that they later can concentrate on running with the ball between players. These drills, in which information is reduced, constitute a useful initial step before progression to more realistic and dynamic learning contexts (12).

To some extent, this emphasis on drills in training design and performance evaluation testing in team games has been unduly influenced by current trends in performance analysis (38). It has been argued that performance analysis remains overly preoccupied with cataloging and grouping discrete performance behaviors and fitness indices (20), with few theoretical models of the performance context in which functional actions emerge (38). For instance, common methods of match or training analysis often rely on recording the frequency of categories of action, such as the number and types of passes performed or distance run by an individual. An important criticism of such methods is that they are somewhat reductionist, failing to enhance understanding of the functional utility of specific actions in subphases of team games (38).

To exemplify the influence of performance analysis in team sports training, empirical observations that elite footballers typically demonstrate a higher rate of recovery after short bouts of sprinting, compared with less skilled individuals $(2,8)$, have resulted in the design of training drills aimed at improving speed endurance. Similar ideas exist for acquisition of movement skill through repetitive deliberate practice programs (43).
However, these types of drills, designed to improve physiological or technique performance, tend to isolate an action from the performance context (creating what has been termed the "closed" environments) and may not allow functional performance behaviors to emerge during interpersonal interactions of players (in more "open" environments) (25). Competitive team games are unpredictable, noisy, dynamic performance environments in which information sources rarely are assured in advance and emergent actions are highly context dependent $(36,38)$. Some coaches use an extensive period of practice drills followed by small-sided and conditioned games (SSCG), consistent with the simple-to-complex principle that emanates from the information processing approach; however, our empirical work has questioned the need for such an extensive focus on drills, implying that training in SSCG can result in a more functional acquisition of performance behaviors $(10,26,39)$.

To summarize, attempting to prepare for team games performance through repetitive practice of isolated and discrete movement patterns is a reductionist approach that is an adequate preparation for producing consistent movement patterns in predictable performance environments. In line with past work, our research program on team games preparation has reinforced the need for practice to, instead, represent the unstable, dynamic, and unpredictable nature of competitive team games. We have observed that training should consist mainly of recreating simulations of the game by not only manipulating practice areas (e.g., width and length of fields) but also the objectives and rules of play (through conditioned games). In this regard, research on SSCG has increased in recent years. Early research on SSCG mainly focused on investigating effects of different task constraints (e.g., number of players involved in each team, field dimensions of practices pitches, and rules of the games) on physiological conditioning during training (18). More recently, and aligned with these advances in physiological preparation for team games, we have argued that the science of team games performance can benefit from the development of a theoretical rationale to describe and explain psychological functioning, such as perception, cognition, movement coordination, and decision making during team game performance (38).

\section{ECOLOGICAL DYNAMICS: A THEORETICAL RATIONALE FOR TEAM GAMES PREPARATION}

Ecological dynamics is an increasingly influential theoretical approach proposing how individual players and sports teams can be modeled as complex social systems, which are inherently nondeterministic (not completely predictable). Such social neurobiological systems exhibit patterns of coordination at a global system level (movement patterns in individuals and interpersonal interactions at a team level in the context of sport performance). These coordination tendencies continuously emerge from ongoing interactions between system components (within and between individual players). Principles governing component interactions in complex systems are based on use of local information, without reference to global system patterns (28).

In our studies, self-organization tendencies have been observed within actions of individuals and also in interpersonal 
interactions between players in different subphases of team sports (e.g., one vs one or two vs one) $(5,23,24)$. In team games, attackers and defenders have been identified as coadapting components of complex self-organizing systems whose continuous interactive behaviors are regulated by information from the relative positioning and timing of movements of teammates and opponents with respect to key task constraints (e.g., goal location, performance area markings, and the ball). Coadaptive interactions between individuals in invasion team games can lead to emergence of spontaneous pattern-forming dynamics and contextual dependency of decisions, which can constrain (and be constrained by) behaviors of teammates and opponents during performance. These theoretical insights provide a theoretical rationale for implementation of SSCG in training programs for team games performance because they facilitate players gaining experience in exploiting inherent self-organizing tendencies and in acquiring skill in coupling actions and decisions to changing informational constraints of competitive performance environments.

A starting point in our research program has been to identify key constraints on emergence of movement behaviors and decisions of individuals in team games performance, so that they can be simulated in SSCG, providing training programs with representative design (30). Our research has revealed how goal-directed behaviors emerge under specific interacting constraints of the specific task, each individual and environmental properties manipulated in different experimental contexts. In interpreting the main findings from this ongoing program of work, it is worth noting that a key aim is to avoid providing specific instructions in experiments to prescriptively constrain participant behaviors. Self-organizing tendencies result in behaviors emerging from participants over time, with little direct external influence (e.g., feedback from an experimenter or coach) and which are sustained by the information flow created by continuous interactions between players in game subphases.

Information in team sports changes instantaneously, and ecological dynamics reveals how performers perceive properties of performance environments as opportunities to act (i.e., affordances) (16). Affordances capture the fit between individual performer constraints and relevant properties of specific performance environments. Perceiving an affordance is to perceive how one can act under specific performance conditions, which need to be simulated carefully in training tasks. In team sports, we have observed that individuals couple actions to relevant properties of particular performance environments, such as the distance to a teammate/opponent (35), goal, or target area $(35,40)$ and location of the ball relative to a teammate/opponent (34). Affordances are dynamic, continuously changing across ongoing performer-performer(s) interactions, providing the rationale for a more extensive focus on SSCG in team games training.

Affordances could be designed into training tasks by faithfully sampling key properties of performance environments. Athletes can learn how to guide their actions according to the informational specificity of each performance context. Our work on movement behavior has demonstrated that, when participants are required to perform actions under controlled laboratory conditions, in which affordances differed from a performance environment, observed patterns of movement coordination significantly differed (29). For example, when cricketers batted against a bowler, ball projection machine, or a video simulation of a bowler, significant variations in timing of movement initiation and downswing initiation were observed (29).

\section{EXEMPLAR RESEARCH OF INFORMATION-BASED PERFORMANCE IN TEAM SPORTS}

Our research on dynamical properties of sports teams (e.g., basketball, association football, and rugby union) has revealed the spatial-temporal interactions between an attacker and defender performing in a dyadic (one vs one) system and key tasks constraints, such as ball trajectory, goal location, and angles to goal target areas. These features of competitive performance environments interact to influence emergence of stability (i.e., coordination between competitors), variability (i.e., loss of coordination and emergence of different modes of coordination), and emergence of new states of organization in attackerdefender relations during performance (38). For example, when an attacker with the ball in rugby union was required to score a try against a defender, our observations revealed phases of competitive performance characterized by periods of equilibrium between the attacker and defender (reflecting a stable state of dyadic system organization, with each player remaining a specific distance apart during performance) (24). During such periods of system stability, competing players tended to adjust their positioning relative to each other and the try line, based on existing and emergent informational constraints. As performance unfolded, these dyadic systems sometimes transited far from this zone of stability. We observed that decreasing values of a key system variable, the interpersonal distance between competing players, can move an attacker-defender dyadic system to critical performance regions (22). Interacting with these critical interpersonal distance values, the relative velocity between competing players in an attacker-defender dyad, moved the competing system toward specific performance outcomes, such as a try being scored by the attacker, destabilizing the temporarily formed dyadic system (23). Our work showed that individual players were pulled toward (attracted to) a specific behavioral solution when a fit emerged between the environmental and individual constraints. Findings illustrated how competing players harnessed movement variability in attempts to destabilize a dyadic system and gain an advantage over an opponent through continuous nonlinear interactions. SSCG can be designed to provide extensive opportunities to experience ongoing interpersonal interactions at critical and specific values of interpersonal distance and relative velocity during training. SSCG played on-field are useful for helping performers attune to action-relevant information and explore ways to achieve key performance outcomes (maintain or eliminate dyadic system stability). Moreover, our work has shown that teammates are attuned to information on opportunities for action for other players, establishing the basis of teamwork. Perception of affordances for teammates sustains communication and interactions at an ecological level of analysis, providing an alternative explanation for shared cognitions to underpin interpersonal coordination from a team's perspective (26). 
In follow-up work, we confirmed how functional interpersonal interactions between attackers and defenders, based on variations in key task constraints, such as relative spatial positioning between attackers, defenders, the ball, and goal during performance, can shape skill performance and decisionmaking behaviors $(21,40)$. We established how emergent actions and decision making of players can be modulated in SSCG by manipulating specific practice task constraints such as the on-field location for dyadic system interactions (17), scaling performer physical characteristics such as height (5), changing initial distances between defenders or between defenders and attackers $(7,21)$, manipulating instructional constraints on players (conservative or risk-taking instructions) (5), and modifying playing rules (27).

These studies have shown that coadaptive behaviors between players involved in SSCG are important inherent processes in team sports that can be harnessed during training when individuals are provided with opportunities to continuously adjust their actions relative to emerging behaviors of others. Coadaptation processes have been demonstrated by spatial and temporal reorganization of small subgroups of attackers or defenders working together to achieve common performance goals. For instance, investigation of a two versus one subphase in rugby union matches by Correia et al. (6) showed how decision making was constrained by the nature of the spatial-temporal relationships between a player passing the ball, a marking defender, and a ball receiver. Positional data of each individual's movement displacement trajectories were analyzed to identify spatial and temporal performance variables, including (i) pass distance (differences between positions of the passer at the moment of pass initiation and ball receiver at the moment of pass reception) and (ii) pass duration (ball flight time from the moment of pass initiation to the moment of ball reception). They calculated the temporal value required for the distance between an attacker and defender to be reduced to zero (assuming that players maintained a constant running velocity) at discrete moments during performance, including (i) when the ball passer first received the ball and (ii) when the ball passer released the ball (Fig. 1). Mean time to contact when the defender was approaching the attacker also was recorded.

Findings revealed that initial values of time to contact when a passer received the ball were related to values for ensuing pass distance and pass duration. Time to contact at that initial performance point also predicted $64 \%$ of the variance observed in the duration of resulting passes. Data revealed that information on time remaining for a ball passer to be tackled by a marking defender at the instant that he or she received the ball acted as a spatial-temporal variable that guided decisions and actions in that two versus one subphase of rugby union. Threshold values of time to contact at critical instances during performance seemed to act as an informational constraint on the type of pass that emerged from skilled rugby union players' interpersonal interactions, that is, they afforded only specific types of passes to emerge (long or short). Establishing this type of perception-action coupling relation is a distinct benefit of training in SSCG.

Passos et al. (28) sought to identify variables that specified functional information to sustain coupling tendencies between attacking teammates in another study of performance in rugby union involving an attacking group of four teammates against two pairs of opponents forming a first and second defensive line. In this experiment, no specific instructions were provided to participants on how to achieve their tactical aims of scoring or preventing a try so that spontaneous interpersonal interactions between teammates could be observed. Data revealed a tendency for attacking players to display functional coadaptive behaviors, leading to a change in the range of interpersonal distance values within the attacking subunit from 2 to $4 \mathrm{~m}$ before the defensive line to 3 to $5 \mathrm{~m}$ of interpersonal distance "inside" the defensive lines. Changes in interpersonal coordination patterns caused by these adaptive behaviors were captured, with running correlation values oscillating between 1 and -1 (between 4 and 10 s; Fig. 2).

Increasing proximity to defensive lines demanded coadaptive behaviors leading to changes in the attackers' relative
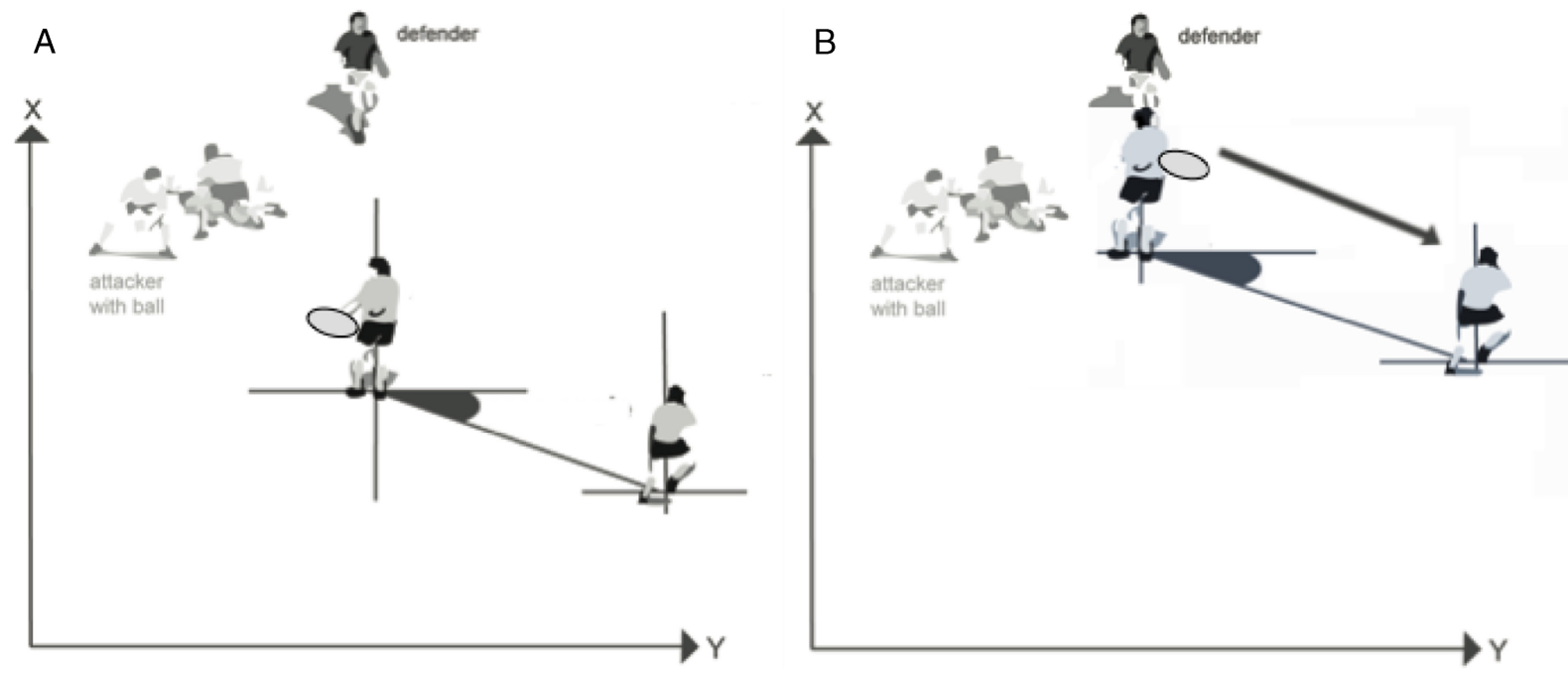

Figure 1. Moments during performance: (A) when the ball passer first received the ball and (B) when the ball passer released the ball. [Adapted from (6). Copyright (C) 2011 Elsevier. Used with permission]. 
positioning and roles within the subunit. This observation signified how the strength of coupling between individuals within a subunit can be decreased, as quantified by $r^{2}$ values. These data revealed high coupling tendencies within most attackerattacker interactions possibly caused by all players running toward the try line at the same time (their actions were constrained by the first coaching principle for the game: to advance upfield).

In addition to such experimental manipulations, we also have observed the dynamics of interpersonal interactions during competitive performance in five versus five futsal games involving 71 individuals from five different national teams. Vilar et al. (37) investigated (i) how location of the goal and ball constrained coordination tendencies of attacker-defender dyadic systems and (ii) how information from the positioning of defenders constrained coordination between a ball carrier and supporting teammates (Fig. 3). Movement displacement trajectories of players and ball were digitized from 52 outfield attacker-defender dyadic system interactions, of which 13 involved ball possession by an attacking player. Relative phase was used as a measure to express the coordination tendencies in the attacker-defender dyadic systems (in-phase or symmetry, 0 degrees; antiphase or antisymmetry, 180 degrees). This is an important measure reflecting whether two dynamical system components are changing synchronously or asynchronously in a performance environment.

Results revealed a stable in-phase pattern of coordination emerging between values of the attacker's distances to defenders and the goal (19\%). Stable patterns of coordination of -60 degrees emerged between values of the attacker's distances to the defenders and the ball (18\%). Finally, in-phase patterns of coordination emerged between distances of the ball carriers to defenders and teammates ( $14 \%$ at 0 degrees). These findings suggest that defenders used information on the attackers' distances to the goal and ball to synchronously adapt their distances to attackers. When an attacker approached the goal or ball, increasing the potential for scoring a goal, the defender moved toward the attacker. Concomitantly, attacking teammates were observed to perceive a higher risk of the ball carrier losing the ball when a defender approached him. In such environmental conditions, teammates were shown to decrease their distances to ball carriers possibly to afford a passing opportunity. These results showed how teammates' actions were constrained by perception of affordances for defenders.

Finally, a case study of competitive 11 versus 11 professional association football by Duarte et al. (13) investigated changes in the magnitude and the structure of performance variability in sports teams as collective systems during a competitive match between two professional teams. Based on raw positional data obtained with the ProZone ${ }^{\circledR}$ tracking system, five compound motion measures were analyzed to investigate collective performance in each team, including surface area, geometrical center, team length, team width, and stretch index. These collective measures are unique in that they revealed significant differences between the structural organization
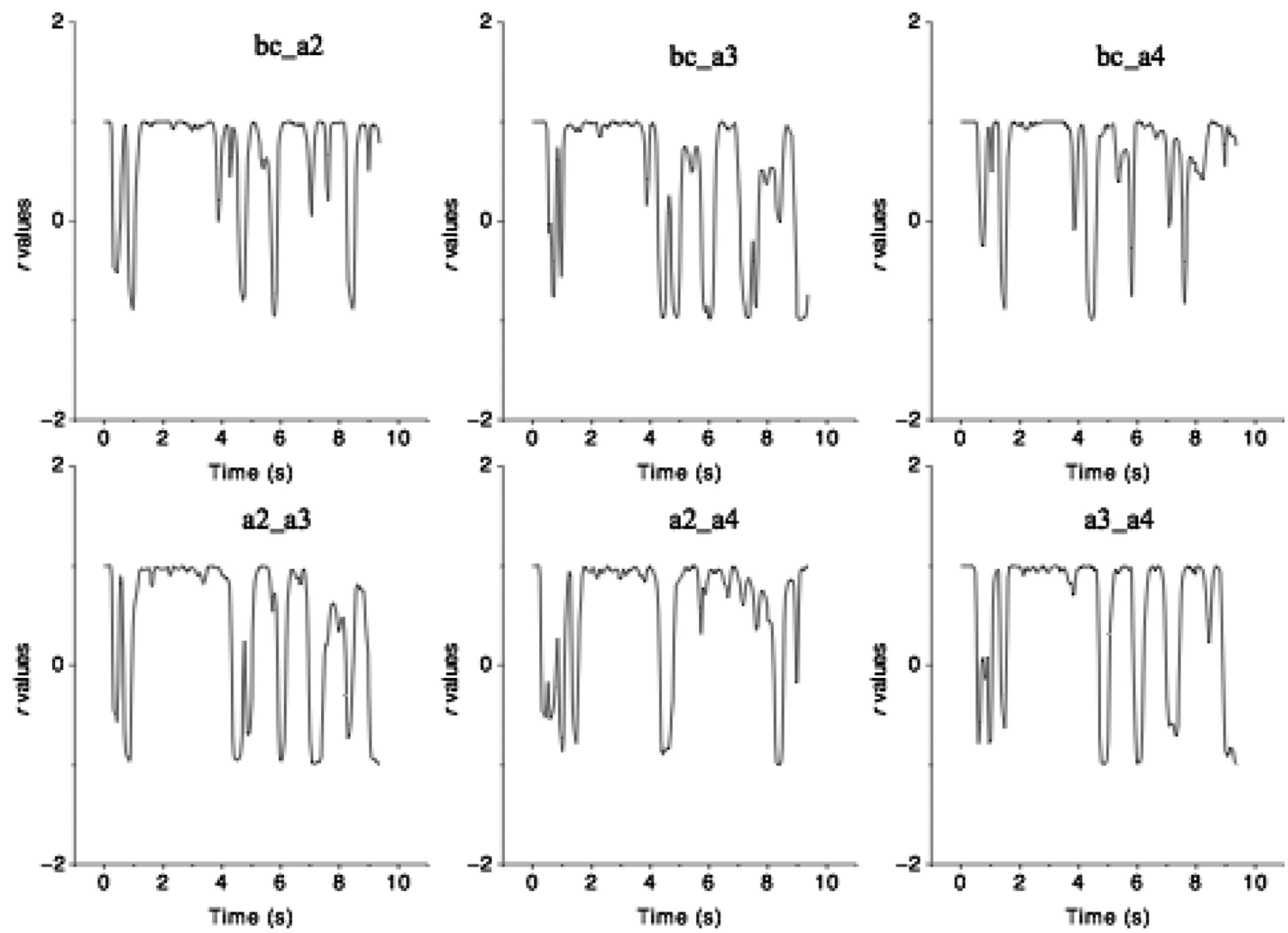

Figure 2. Running correlation values capturing adaptive interpersonal coordination tendencies during phases of attacker-defender interactions. (Reprinted from (28). Copyright (C) 2011 Taylor \& Francis. Used with permission). 

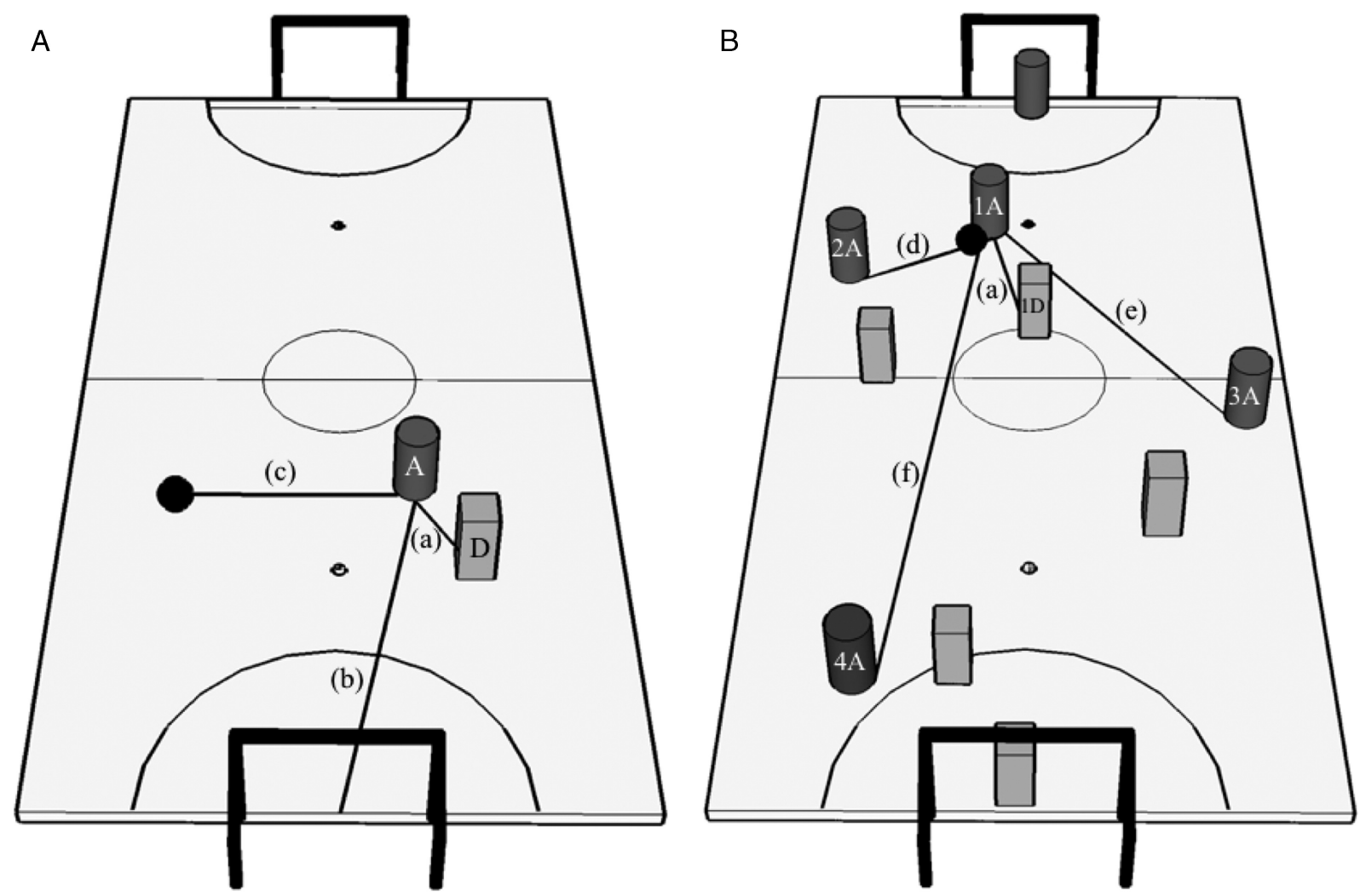

Figure 3. How information from the location of the ball and goal and positioning of opponents constrains coordination tendencies between ball dribbler and teammates: $(A)(a)$ interpersonal distance between the attacker $(A)$ and the nearest defender $(D),(b)$ distance of the attacker $(A)$ to the centre of the goal, and (c) distance of the attacker $(A)$ to the ball; $(B)(a)$ interpersonal distance between the ball carrier (1st attacker or 1A) and marker (1D), $(d)$ distance of the ball carrier (first attacker or $1 A$ ) to the second attacker $(2 A)$, (e) distance of the ball carrier $(1 A)$ to the third attacker $(3 A)$, and $(f)$ distance of the ball carrier $(1 A)$ to the fourth attacker $(4 A)$.

of full teams across different periods of a competitive match. Approximate entropy values showed a significant and large decreasing effect during each half in the structure of performance variability. Data showed that, when attacking, both teams attempted to cover a greater area of the pitch and displayed a greater dispersion of players, greater length and width values, and a greater distance of the team's geometrical center from the center of the field. A key point is that these data concur with reports of a superior percentage of ball possession for top football teams playing at home than for visiting teams (19). Teams also seemed to become more regular and predictable in their organizational shape over the course of a match perhaps because of factors such as increasing fatigue and performance-related stress (32). The implication of this study of 11 versus 11 association football during elite competition is that training design using SSCG could aim to help players develop similar structural organizational tendencies (e.g., adopting functional stretch index and geometric values) when practicing offensive and defensive patterns of play.

\section{APPLICATIONS OF SSCG IN PEDAGOGICAL PRACTICE}

Our experimental and observational research on team games has helped practitioners to better understand how emergent behaviors can be constrained through design and manipulation of constraints in SSCG. Small-sided and conditioned games can be used to help learners gain experience in picking up specifying (functionally relevant) information for continuously regulating interpersonal interactions with teammates and defenders during performance. In training, subtasks of the games can be simulated by using SSCG because this method involves manipulating key constraints to facilitate rapid discovery of functional movement behaviors (4). This body of work suggests that SSCG can simulate specific subphases of team games such as one versus one, two versus one, four versus two, and four versus four because they amplify information for action by providing increased frequency of opportunities for interpersonal interactions to occur between attackers and defenders (15). SSCG as subphase simulations contain functional levels of contextual variability, which provide learners with numerous occasions to adapt their actions and decisions through continuous ongoing interactions with teammates and opponents and other task constraints (such as the goal, ball, and field markings). Instead of learners practicing static drills or playing in full-sided games in major team sports like soccer or basketball, manipulation of objectives and/or rules in SSCG can increase opportunities for common attacking and defensive subphases to emerge frequently. They also can be used to encourage breaking of stability in dyadic systems through dribbling skills or maintaining system stability through ball possession or defensive positioning. The structure and organization of SSCG need to be designed specifically for individual learners to practice exploring different performance solutions as performance environment contexts change, rather than practicing the same actions repetitively. Rather than 
encouraging learners to dribble a ball around cones, pass in straight lines to each other, tackle static bags, or shoot a stationary ball in drills, the task constraints of SSCG can be manipulated to encourage coadaptive movement behaviors through facilitating continuous interpersonal interactions of learners with teammates $(1,23,24)$, opponents (7), and key field markings (14). Manipulation of task constraints during SSCG is extremely important to prevent randomness in the actions of learners: their exploratory activities need to be bounded to encourage them to explore the balance between stable and variable movement patterns in achieving specific task goals. Emphasis on exploratory learning within SSCG is a prerequisite for acquiring skill in interpersonal interactions (4). In such simulated performance environments, learners need to be able to identify specific information sources that they can become attuned to and use to regulate behavior.

A nonlinear pedagogy is an important approach to facilitate the emergence of decision-making behaviors and functional movement patterns by simulating competitive performance interactions between performers and environmental constraints (4). This aim can be achieved through manipulation of important variables in SSCG during training to create conditions for interpersonal interactions, which simulate those in competitive performance. These SSCG manipulations can be related to (i) task objectives (score or maintain ball possession) and (ii) rules of play: space (e.g., the nature of the playing surface, playing area dimensions and shape), time (e.g., time span of matches, time to attain a subobjective of the match), players (e.g., number of players in each team, number of teams, roles of players), equipment (e.g., size and number of goals and balls), and intrapersonal and interpersonal coordination (e.g., limbs allowed to contact the ball or players allowed to pass the ball). For example, learners playing SSCG to simulate $3 \mathrm{v} 3$ or $3 \mathrm{v} 1$ subphases of the game on a small pitch are afforded more opportunities for passing, shooting, and dribbling, resulting in an amplified exposure to information that regulates actions. In addition, such subsystems operate closer to instability, with fewer opportunities to settle into stable states of organization because each player in an SSCG is continuously influencing system organization. In full-sided team games, the global system is harder to destabilize because some subgroups on field or court can be inactive. These inherent instability tendencies in SSCG are useful for learners to gain more experience in supporting teammates with the ball, intercepting the ball to regain possession when defending, and coordinating with teammates to deny space and prevent shooting opportunities (11). SSCG also can increase the amount of time spent in playful and enjoyable activities by allowing learners to experience simulations of competitive team games.

\section{CONCLUSIONS}

Findings from our research program have provided theoretical advances that can benefit coaches, sport scientists, pedagogues, sport performance analysts, and physical educators in learning and structuring practice designs in team sports. The focus is on creating simulated versions of major games that capture interpersonal interactions required for competitive team games performance, predicated on specifying information sources, which underpin coordination tendencies between an athlete/ group of athletes and the environment. Our research in ecological dynamics suggests that skill acquisition tasks and evaluation tests in sport development programs need to be predicated on performance in SSCG rather than isolated practice drills in static practice contexts.

\section{Acknowledgments}

No funding was received by any of the authors for the production of the studies described in this research program or the article submitted. None of the authors have professional relations with commercial companies or business interests that may be of relevance to the studies described in this article.

\section{References}

1. Araújo D, Davids K, Hristovski R. The ecological dynamics of decision making in sport. Psychol. Sport Exerc. 2006; 7:653-76.

2. Bangsbo J, Iaia FM, Krustrup P. The Yo-Yo intermittent recovery test: a useful tool for evaluation of physical performance in intermittent sports. Sports Med. 2008; 38:37-51.

3. Chow J, Davids K, Button C, Koh M. Variation in coordination of a discrete multiarticular action as a function of skill level. J. Motor Behav. 2007; 39:463-79.

4. Chow J, Davids K, Button C, Shuttleworth R, Renshaw I, Araújo D. The role of nonlinear pedagogy in physical education. Rev. Educ. Res. 2007; 77:251-78.

5. Cordovil R, Araújo D, Davids K, et al. The influence of instructions and body-scaling as constraints on decision-making processes in team sports. Eur. J. Sport Sci. 2009; 9:169-79.

6. Correia V, Araújo D, Craig C, Passos P. Prospective information for pass decisional behavior in rugby union. Hum. Mov. Sci. 2011; 30:984-97.

7. Correia V, Araújo D, Duarte R, Travassos B, Passos P, Davids K. Changes in practice task constraints shape decision-making behaviours of team games players. J. Sci. Med. Sport. 2012; 15:244-9.

8. Coutts AJ, Rampinini E, Marcora SM, Castagna C, Impellizzeri FM. Heart rate and blood lactate correlates of perceived exertion during smallsided soccer games. J. Sci. Med. Sport. 2009; 12:79-84.

9. Davids K, Araújo D. The concept of "Organismic Asymmetry" in sport science. J. Sci. Med. Sport. 2010; 13:633-40.

10. Davids K, Araújo D, Hristovski R, Passos P, Chow JY. Ecological dynamics and motor learning design in sport. In: Williams AM, and Hodges N, editors. Skill Acquisition in Sport: Research, Theory $\mathcal{E}$ Practice. London: Routledge; 2012. p. 112-30.

11. Davids K, Chow JY, Shuttleworth R. A constraints-based framework for nonlinear pedagogy in physical education. J. Phys. Educ. N. Z. 2005; 38:17.

12. Davids K, Kingsbury D, Bennett S, Handford C. Information-movement coupling: implications for the organization of research and practice during acquisition of self-paced extrinsic timing skills. J. Sports Sci. 2001; 19:117-27.

13. Duarte R. Interpersonal Coordination Tendencies in Soccer. Lisbon (Portugal): LAP Lambert Academic Publishing; 2012.

14. Esteves P, Araújo D, Davids K, Vilar L, Travassos B, Esteves C. Interpersonal dynamics and relative positioning to scoring target of performers in 1 vs 1 sub-phases of team sports. J. Sports Sci. 2012; 30:1285-93.

15. Fenoglio R. The Manchester United 4 v 4 pilot scheme for U-9's part II: the analysis. Insight F. A. Coaches Assoc. J. 2003; 21-4.

16. Gibson J. The Ecological Approach to Visual Perception. Boston (MA): Houghton Mifflin; 1979.

17. Headrick J, Davids K, Renshaw I, Araújo D, Passos P, Fernandes O. Proximity-to-goal as a constraint on patterns of behaviour in attackerdefender dyads in team games. J. Sports Sci. 2012; 30:247-53.

18. Hill-Hass S, Dawson B, Coutts A, Rowsell G. Physiological responses and time-motion characteristics of various small-sided soccer games in youth players. J. Sports Sci. 2009; 27:1-8

19. Lago C, Martin R. Determinants of possession of the ball in soccer. J. Sports Sci. 2007; 25:969-74.

20. McGarry T. Applied and theoretical perspectives of performance analysis in sport: scientific issues and challenges. Int. J. Perform. Anal. Sport. 2009; 9:128-40.

21. Orth D, Davids K, Araújo D, Renshaw I, Passos P. Effects of a defender on run-up velocity and ball speed when crossing a football. Eur. J. Sport Sci. 2012; 0:1-8. 
22. Passos P, Araújo D, Davids K. Self-organization processes in field-invasion team sports implications for leadership. Sports Med. 2013; 43:1-7.

23. Passos P, Araujo D, Davids K, Gouveia L, Milho J, Serpa S. Informationgoverning dynamics of attacker-defender interactions in youth rugby union. J. Sports Sci. 2008; 26:1421-9.

24. Passos P, Araújo D, Davids K, et al. Interpersonal pattern dynamics and adaptive behavior in multiagent neurobiological systems: conceptual model and data. J. Motor Behav. 2009; 41:445-59.

25. Passos P, Araujo D, Davids K, Shuttleworth R. Manipulating constraints to train decision making in rugby union. Int. J. Sports Sci. Coach. 2008; 3:125-40.

26. Passos P, Cordovil R, Fernandes O, Barreiros J. Perceiving affordances in rugby union. J. Sports Sci. 2012; 30:1175-82.

27. Passos P, Davids K, Araújo D, Paz N, Minguens J, Mendes J. Networks as a novel tool for studying team ball sports as complex social systems. J. Sci. Med Sport. 2011; 14:170-6.

28. Passos P, Milho J, Fonseca S, Borges J, Araújo D, Davids K. Interpersonal distance regulates functional grouping tendencies of agents in team sports. J. Motor Behav. 2011; 43:155-63.

29. Pinder R, Davids K, Renshaw I, Araújo D. Manipulating informational constraints shapes movement reorganization in interceptive actions. Atten. Percept. Psychophys. 2011; 73:1242-54.

30. Pinder R, Davids K, Renshaw I, Araújo D. Representative learning design and functionality of research and practice in sport. J. Sport Exerc. Psychol. 2011; 33:146-55.

31. Pinder R, Renshaw I, Davids K. Information-movement coupling in developing cricketers under changing ecological practice constraints. Hum. Mov. Sci. 2009; 28:468-79.

32. Royal K, Farrow D, Mujika I, Halson SL, Pyne D, Abernethy B. The effects of fatigue on decision making and shooting skill performance in water polo players. J. Sports Sci. 2006; 24:807-15.
33. Tessitore A, Meeusen R, Piacentini MF, Demarie S, Capranica L. Physiological and technical aspects of "6-a-side" soccer drills. J. Sports Med. Phys. Fitness. 2006; 46:36-43.

34. Travassos B, Araújo D, Davids K, Vilar L, Esteves P, Correia V. Informational constraints shape emergent functional behaviours during performance of interceptive actions in team sports. Psychol. Sport Exerc. 2012; 13:216-23.

35. Travassos B, Araújo D, Duarte R, McGarry T. Spatiotemporal coordination behaviors in futsal (indoor football) are guided by informational game constraints. Hum. Mov. Sci. 2012; 31:932-45.

36. Travassos B, Duarte R, Vilar L, Araújo D, Davids K. Practice task design in team sports: Representativeness enhanced by increasing opportunities for action. J. Sports Sci. 2012; 30:1447-54.

37. Vilar L. Informational Constraints on Performance in Futsal (Indoor Football). Lisbon (Portugal): Lambert Academic Publishing; 2012.

38. Vilar L, Araújo D, Davids K, Button C. The role of ecological dynamics in analysing performance in team sports. Sports Med. 2012; 42:1-10.

39. Vilar L, Araújo D, Davids K, Renshaw I. The need for "representative task designs" in evaluating efficacy of skills tests in sport: a comment on Russell, Benton and Kingsley (2010). J. Sports Sci. 2012; 30:1727-30.

40. Vilar L, Araújo D, Davids K, Travassos B. Constraints on competitive performance of attacker-defender dyads in team sports. J. Sports Sci. 2012; 30:459-69.

41. Wickens CD. Attention and skilled performance. In: Holding DH, editor. Human Skills. Chichester: John Wiley; 1989, p. 71-106.

42. Wickens CD. Engineering Psychology and Human Performance. Champaign (IL): Harper Collins; 1997.

43. Williams AM, Ericsson K. Perceptual-cognitive expertise in sport: some considerations when applying the expert performance approach. Hum. Mov. Sci. 2005; 24:283-307. 